\section{Life: perhaps we should take the porridge theory with a pinch of salt}

SIR - In his Book Review of The Goldilocks Enigma ("Life in the universal porridge" Nature 444, 423-424; 2006), Jim Al-Khalili discusses Paul Davies' answers to the question "Why is the Universe just right for life?".

The question relies upon the widely held assumption that life, in particular human life, is a unique form of matter requiring a special explanation for its existence.

As a biologist, I agree that life is a fascinating phenomenon, and humans may well be among the most complex entities in the Universe.

However, all past and present life-forms on Earth have been the products of one particular evolutionary process, which was driven by natural selection and which operates on the basis of specific genetic mutations and selective environments. If these mutations and environments had been different, the products of this evolutionary process would also have differed. Indeed, the organisms we know of, including our own species, might never have evolved at all.

Thus, our existence does not require a special category of explanation, beyond natural selection. To think so can be seen as a form of chauvinism - seductive but misguided - that elevates the specific aggregates of matter on this planet, and their historically conditioned features, to an inappropriately lofty status.

This argument also applies to SETI, the search for extraterrestrial intelligence, which is based on the same tempting assumption. Life, consciousness and intelligence, as we know them, are highly unlikely to be found elsewhere in the Universe, nor are they likely to be inevitable consequences of any sufficiently 'advanced' darwinian system. Rather, they are specific outcomes of one particular process of evolution, occurring on one of the billions of planets in this Universe. (If the Multiverse exists, there could be other universes almost identical to ours, and therefore there could be other life-forms like us, but presumably we would not be able to communicate with them.)

These conclusions will be disappointing to many - as they are, sometimes, to me. But, as Richard Dawkins commented about people who have been disturbed by his book The Selfish Gene, in his Darwin@LSE public lecture in London this year, "If something is true, no amount of wishful thinking can undo it".

Rather than leaving these matters, as Davies suggests, to "philosophers and priests", or to physicists, perhaps we should listen to the biologists as well.
Then some of these questions might be considered less troubling, and less important, than they currently seem to be.

Peter Wigley

22 Chatham Road,

Keswick, South Australia 5035, Australia

\section{Life: porridge would be just right for each universe}

SIR — Jim Al-Khalili's Book Review of Paul Davies' The Goldilocks Enigma ("Life in the universal porridge" Nature 444, 423-424; 2006) explains with great clarity why the anthropic principle is an incomplete answer to the great question of 'Life, the Universe and everything', and why the concept of a Multiverse is more satisfactory.

However, there is an alternative - even if we assume that the only way to make a different universe is to change a few fundamental constants.

Arguments in favour of fine-tuning typically show that some key ingredient of our current Universe, such as atoms or stars, becomes unstable if some physical constant is changed by a relatively small amount and therefore cannot exist in a universe with different constants. But in such circumstances, what is interesting is not the instability of some particular state, but what the system does instead. If conventional atoms or stars become unstable, what other organized forms of matter might arise? In particular, which values of the physical constants permit structures complex enough to resemble intelligent life?

It is not easy to answer this question. At the moment, we cannot rigorously deduce the structure of the helium atom from basic physics, let alone that of a living organism. Our understanding of atomic structure depends on various ad hoc simplifications, justified by comparison with observation. This approach is not available for universes with different constants, so we have no real idea how they might behave.

If - as experience with simpler dynamical systems suggests - large regions of universespace permit complex structures, then our Universe actually has a reasonable chance of falling into such a region, and the complex structures that arise will necessarily be consistent with the laws of that universe.

The real message of Goldilocks is not that Baby Bear's porridge was 'just right'. It was just right for Goldilocks — but cold porridge was also just right for Mummy Bear and hot porridge was just right for Daddy Bear. We are the product of our own universal porridge, and it will always be just right for us.

lan Stewart

Mathematics Institute, University of Warwick, Coventry CV4 7AL, UK

\section{Biography of Crick aims to inspire a wider audience}

SIR - We do hope that your readers are not put off reading Matt Ridley's biography Francis Crick: Discoverer of the Genetic Code (HarperCollins, 2006) by Horace Judson's review (Nature 443, 917-918; 2006). Ridley's book, which, as one of a series of biographies, had to conform in length to a standard, makes no pretence to be the academic biography that Judson was apparently expecting. He should know (as Ridley does, and makes clear in his acknowledgements) that the 'definitive' biography of Crick is being written by Bob Olby. Ridley set out to write about 'the greatest biologist of the twentieth century' for a wide audience.

Judson comments, for example, that insufficient detail was given about Crick and Watson's interactions with the Wilkins and Franklin group. But these interactions have been considered by historians many times, including by Judson himself in his book The Eighth Day of Creation (Simon \& Schuster, 1979), and could only have been condensed in this brief account.

Ridley conveys, in a way that is both clear and affectionate, Crick's unique mix of fun and genius, as well as the highlights of his scientific achievements. Judson calls Ridley a 'journalist'. That's an arguable description, but in a world where the communication of science to a general audience is so essential, we wish there were more 'journalists' like him. His biography of Crick will make many eager to read more about this inspiring man. Michael Ashburner ${ }^{\star}$, Mark Bretscher $r$,

Peter A. Lawrence:

*Department of Genetics,

†MRC Laboratory of Molecular Biology,

tDepartment of Zoology,

University of Cambridge, Cambridge, UK

\section{Shellfish view of omega-3 and sustainable fisheries}

SIR - The mackerel pictured in A. K. Duttaroy's review of Susan Allport's book Queen of Fats (Nature 444, 425; 2006) are indeed a source of omega-3s, but others are just as good and more sustainable. Early humans evolved eating fish, probably intertidal shellfish, while living a shoreline existence in Africa. They didn't have boats to trawl for pelagic oily fish such as mackerel, nor did they have hooks and lines. Now, as then, inter-tidal herbivorous shellfish such as mussels and clams can help people reach a healthy 1:1 balance of omega- 3 and omega- 6 fats in their diets, instead of the current 1:17. Anthony Robson Institute of Environmental Sustainability, Swansea University, Singleton Park, Swansea SA2 8PP, UK 